This is an electronic reprint of the original article. This reprint may differ from the original in pagination and typographic detail.

Author(s): Suhonen, Jouni

Title: Impact of the quenching of gA on the sensitivity of $0 v \beta \beta$ experiments

Year: $\quad 2017$

Version:

Please cite the original version:

Suhonen, J. (2017). Impact of the quenching of gA on the sensitivity of Ov $\beta \beta$ experiments. Physical Review C, 96(5), Article 055501. https://doi.org/10.1103/physrevc.96.055501

All material supplied via JYX is protected by copyright and other intellectual property rights, and duplication or sale of all or part of any of the repository collections is not permitted, except that material may be duplicated by you for your research use or educational purposes in electronic or print form. You must obtain permission for any other use. Electronic or print copies may not be offered, whether for sale or otherwise to anyone who is not an authorised user. 


\title{
Impact of the quenching of $g_{A}$ on the sensitivity of $0 v \beta \beta$ experiments
}

\author{
Jouni Suhonen \\ University of Jyvaskyla, Department of Physics, P.O. Box 35, FI-40014 Jyvaskyla, Finland
}

(Received 5 July 2017; published 27 November 2017)

\begin{abstract}
Detection of the neutrinoless $\beta \beta(0 \nu \beta \beta)$ decay is of high priority in the particle- and neutrino-physics communities. The detectability of this decay mode is strongly influenced by the value of the weak axial-vector coupling constant $g_{A}$. The recent nuclear-model analyses of $\beta$ and $\beta \beta$ decays suggest that the value of $g_{A}$ could be dramatically quenched, reaching ratios of $g_{A}^{\text {free }} / g_{A} \approx 4$, where $g_{A}^{\text {free }}=1.27$ is the free, neutron-decay, value of $g_{A}$. The effects of this quenching appear devastating for the sensitivity of the present and future $0 v \beta \beta$ experiments since the fourth power of this ratio scales the $0 v \beta \beta$ half-lives. This, in turn, could lead to some two orders of magnitude less sensitivity for the $0 \nu \beta \beta$ experiments. In the present article it is shown that by using a consistent approach to both the two-neutrino $\beta \beta$ and $0 v \beta \beta$ decays by the proton-neutron quasiparticle random-phase approximation, the feared two-orders-of-magnitude reduction in the sensitivity of the $0 v \beta \beta$ experiments actually shrinks to a reduction by factors in the range 2-6. This certainly has dramatic consequences for the potential to detect the $0 \nu \beta \beta$ decay.
\end{abstract}

DOI: 10.1103/PhysRevC.96.055501

\section{INTRODUCTION}

The nuclear matrix elements (NMEs) of neutrinoless $\beta \beta$ $(0 \nu \beta \beta)$ decay are becoming an increasingly hot issue in present-day neutrino and particle physics since they are the ones that relate the absolute mass scale of the neutrino (the effective neutrino mass $\left.\left\langle m_{v}\right\rangle\right)$ to the constantly increasing sensitivity of the present and future $0 \nu \beta \beta$ experiments [1-3]. The formidable progress in the sensitivity of the neutrinomass related underground experiments is witnessed in the rapidly increasing accuracy in the measured half-lives of the two-neutrino $\beta \beta(2 v \beta \beta)$ decays for some ten nuclear systems $[4,5]$. At the same time the lower limits for the half-lives of the $0 \nu \beta \beta$ decay mode keep steadily increasing [3]. Important nuclei for the present and future $0 \nu \beta \beta$ experiments are ${ }^{76} \mathrm{Ge}$ (the GERDA experiment [6], and in the future GERDA and Majorana [7]), ${ }^{82} \mathrm{Se}$ (the NEMO-3 experiment [8], and in the future SuperNEMO [9] and MOON [10]), ${ }^{96} \mathrm{Zr}$ (the NEMO-3 experiment [11]), ${ }^{100}$ Mo (the NEMO-3 experiment [12], and in the future AMoRE [13], LUMINEU [14], CUPID [15], MOON [10]), ${ }^{116} \mathrm{Cd}$ (the NEMO-3 experiment [16], and in the future AURORA [17], COBRA [18]), ${ }^{130} \mathrm{Te}$ (the CUORE experiment [19], and in the future CUORE and SNO+ [20]), and ${ }^{136} \mathrm{Xe}$ (the EXO [21] and KamLAND-Zen [22] experiments, and in the future NEXT [23] and PandaX-III [24]).

As mentioned above, the exact sensitivity of the present and future $0 \nu \beta \beta$ experiments to the effective neutrino mass $\left\langle m_{v}\right\rangle$ can be determined only if the involved NMEs are known accurately enough. For this reason, more and more attention is being paid to the issue of computing the values of these NMEs in a proper way [25].

The article is organized as follows. In Sec. II the theory background of the $0 v \beta \beta$ is briefly outlined and in Sec. III the background of the $g_{A}$ problem is discussed. In Sec. IV the adopted nuclear model and its properties are introduced, as also the fitting procedure for consistent prediction of the $0 v \beta \beta$ NMEs. In Sec. V the results for the computed half-lives are given and in Sec. VI the final conclusions are drawn.

\section{THEORETICAL BACKGROUND}

By assuming the neutrino-mass mechanism to be the dominant one, one can write the connection between the $0 \nu \beta \beta$ half-life and the involved NMEs conveniently as

$$
t_{1 / 2}^{(0 v)}=F^{(0 v)}\left(g_{A}\right)\left(\left\langle m_{v}\right\rangle[\mathrm{eV}]\right)^{-2},
$$

where $\left\langle m_{v}\right\rangle$ is the effective neutrino mass [1] in units of eV and $F^{(0 v)}$ can be coined reduced half-life:

$$
F^{(0 v)}\left(g_{A}\right)=\left(g_{A}^{2}\left|M^{(0 v)}\right|\right)^{-2}\left(G_{0}^{(0 v)}\right)^{-1}\left(m_{e}[\mathrm{eV}]\right)^{2} .
$$

Here, $m_{e}$ is the electron rest mass in units of $\mathrm{eV}, G_{0}^{(0 v)}$ is the leptonic phase-space factor defined in [26], and $g_{\mathrm{A}}$ is the weak axial-vector coupling constant with the free value $g_{A}^{\text {free }}=1.27$, determined from the decay of a neutron to a proton [27]. This value is to some extent protected in nuclear medium by the PCAC (partially conserved axial-vector current) hypothesis. The $0 \nu \beta \beta$ nuclear matrix element, $M^{(0 v)}$, consists of the Gamow-Teller (GT), Fermi (F), and tensor (T) parts as

$$
M^{(0 v)}=M_{\mathrm{GT}}^{(0 v)}-\left(\frac{g_{\mathrm{V}}}{g_{A}}\right)^{2} M_{\mathrm{F}}^{(0 v)}+M_{\mathrm{T}}^{(0 v)} .
$$

\section{BACKGROUND OF THE $g_{\mathrm{A}}$ PROBLEM}

Whereas the NME $M^{(0 v)}$ of Eq. (3) has been under intensive theoretical discussion for decades, the other involved quantity, $g_{A}$, has remained an innocent bystander, not provoking heated discussions in the $0 \nu \beta \beta$ community. Attempts to deal with the $g_{A}$-quenching problem include the shell-model calculations [28-30] in the $0 p$ and $1 s-0 d$ shells and [31-33] in the $1 p-0 f$ shell and beyond. The review [34] gives a comprehensive account of the $g_{\text {A }}$ problem.

Only recently the possibly decisive role of $g_{A}$ in the half-life (1) and in the discovery potential of the $0 \nu \beta \beta$ experiments has surfaced [35]. In [35] a comparison of the experimental and 


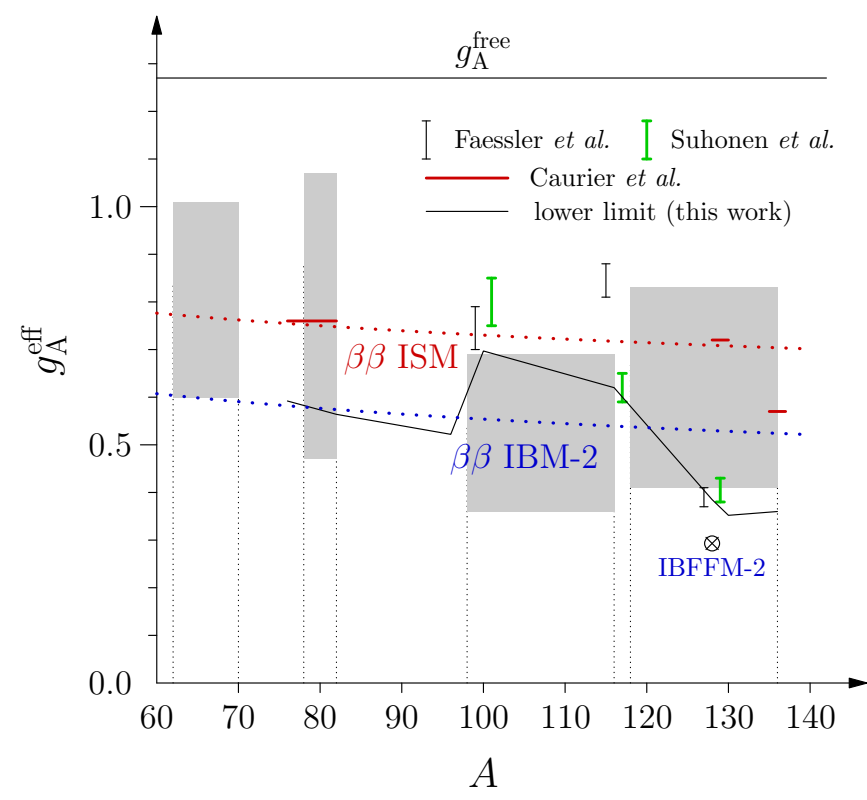

FIG. 1. Effective values of $g_{A}$ in different theoretical approaches for the nuclear mass range $A=62-136$. For more information see the text.

computed $2 v \beta \beta$ half-lives of a number of nuclei yielded the striking result

$$
g_{A}^{\text {eff }}(\mathrm{IBM}-2)=1.269 A^{-0.18} ; \quad g_{A}^{\text {eff }}(\mathrm{ISM})=1.269 A^{-0.12},
$$

where $A$ is the mass number and IBM-2 stands for the microscopic interacting boson model and ISM is an acronym for the interacting shell model. The result (4) implies that strongly quenched effective values of $g_{A}$, in the range $g_{A}^{\text {eff }}=0.5-0.7$ for $A=76-136$, are possible, thus affecting substantially the discovery potential of the $0 \nu \beta \beta$ experiments.

Although the study [35] was the first to draw considerable attention in the experimental $0 v \beta \beta$ community, it was not the first one to point to a possible strong quenching of $g_{A}$. Already the study [36] gave indications of a heavily quenched effective $g_{A}$, in the range $g_{A}^{\text {eff }}=0.39-0.84$. In [36] the Gamow-Teller $\beta$ decays and $2 v \beta \beta$ decays in the $A=100,116,128 \beta \beta$-decay triplets were studied by the use of the proton-neutron quasiparticle random-phase approximation ( $p n \mathrm{QRPA})$, and a least-squares procedure was used to optimize the values of the experimentally over-constrained parameters $g_{A}$ and $g_{\mathrm{pp}}$, where $g_{\mathrm{pp}}$ is the interaction strength of the particle-particle part of the proton-neutron interaction [37,38]. Also in the ISM studies $[39,40]$ very low values of $g_{A}$, in the range $g_{A}^{\text {eff }}=0.57-0.76$, were obtained. Around the same time a similar study as in [36] was carried out in $[41,42]$ with results comparable with those of [36].

Later, the more comprehensive $p n \mathrm{QRPA}$ studies [43-45] of Gamow-Teller $\beta$ decays of a wide range of nuclei $(A=$ 62-142) predicted heavily quenched values of $g_{A}$. These values depend on the mass number $A$ and they are depicted for four separate mass ranges as hatched boxes in Fig. 1. In the same figure, with an obvious notation, also the curves (4) are given, the IBM-2 (ISM) results by a blue (red) dotted curve. Fig. 1 presents also the ISM results of [40] (red horizontal bars), the $p n \mathrm{QRPA}$ results of [36] (thin black vertical bars), and the pnQRPA results of $[41,42]$ (thick green vertical bars). The cross inside a circle presents the result $g_{A}^{\text {eff }}=0.293$ of a $\beta \beta$ analysis of ${ }^{128} \mathrm{Te}$ using the microscopic interacting boson-fermion model (IBFFM-2) [46].

\section{NUCLEAR MODEL AND THE TWO-STAGE FITTING PROCEDURE}

There are many models which have recently been used to compute the $0 \nu \beta \beta$ NMEs: the quasiparticle random-phase approximation (QRPA), in its proton-neutron version ( $p n \mathrm{QRPA}$ ) (see [47] and references therein) and its renormalized extensions [48,49], the interacting shell model (ISM) [50], the microscopic interacting boson model (IBA-2) [51], the Gognybased energy-density functional (EDF) [52], and the projected Hartree-Fock-Bogoliubov mean-field scheme (PHFB) [53]. Very recently also the beyond-mean-field covariant density functional theory [54] and the corresponding non-relativistic version [55] have been used to describe the $0 \nu \beta \beta$ decays of nuclei. For more details see the reviews [25,56].

The $p n \mathrm{QRPA}$ stands out favorably from the rest of the above-listed theoretical approaches by the following features:

(i) The $p n \mathrm{QRPA}$ is the only nuclear model which avoids the use of closure approximation for the mediumheavy and heavy nuclei in the $0 \nu \beta \beta$ calculations. However, there exists a non-closure calculation in the framework of the ISM in [57] for the $0 \nu \beta \beta$ decay of the light nucleus ${ }^{48} \mathrm{Ca}$.

(ii) $p n \mathrm{QRPA}$ can accommodate large single-particle bases, including all the relevant spin-orbit-partner orbitals, in the calculations,

(iii) $p n$ QRPA may fail to predict properties of individual states but the gross features of an energy region of nuclear states can be reliably accounted for by the $p n$ QRPA [58].

The features (i)-(iii) of the $p n$ QRPA make it an ideal nuclear model to combine the $2 \nu \beta \beta$ and $0 \nu \beta \beta$ calculations in a consistent way. Only for light nuclei the single-particle model space is not dense enough for a reliable NME calculation, in particular if the nucleus is doubly magic, like ${ }^{48} \mathrm{Ca}$, since the $\mathrm{BCS}$ procedure used in $p n \mathrm{QRPA}$ framework is not very precise at closed major shells. This is why the $0 \nu \beta \beta$ decay of ${ }^{48} \mathrm{Ca}$ is not included in the present analyses. The above features (i)-(iii) were highlighted in [59] where the $g_{\text {pp }}$ parameter of the $p n \mathrm{QRPA}$ was determined by reproducing the experimental $2 v \beta \beta$ half-life for a given value of $g_{A}$, in that case $g_{A}=g_{A}^{\text {free }}$. This approach was adopted in the follow-up works of [60-62] in order to determine the Hamiltonian parameters as reliably as possible for a consistent prediction of the $0 \nu \beta \beta$ NMEs.

At this point it is fair to state that the ISM is a more complete theory than $p n \mathrm{QRPA}$ in terms of the number of included many-body configurations in a given single-particle model space. It has, at least, some approximate means to accommodate the feature (ii) in the calculations, but the feature (i) is out of reach for the heavier nuclei. The ISM has, however, 
the advantage that also it can produce $2 \nu \beta \beta$ NMEs without resorting to the closure approximation. This means that the analyses of the present work could also be performed in the ISM framework when suitable "particle-particle interaction strength", $g_{\text {pp }}$ (ISM), can be identified and fitted by the $2 \nu \beta \beta$ half-life data through different values of $g_{A}$. In fact, this type of study would be highly welcome to see how robust the results of the present work really are.

The relation of the $p n \mathrm{QRPA}$ Hamiltonian and the $2 v \beta \beta$ decay was further deepened in the work [63] where an isospin-restoration scheme for the $p n \mathrm{QRPA}$ was proposed. There the particle-particle parts of the $p n \mathrm{QRPA}$ matrices were divided into isoscalar $(T=0)$ and isovector $(T=1)$ parts, splitting the particle-particle interaction strength parameter $g_{\mathrm{pp}}$ to its isovector $g_{\mathrm{pp}}^{T=1}$ and isoscalar $g_{\mathrm{pp}}^{T=0}$ components. The two strength parameters can be adjusted separately since they are practically independent of each other. The isovector parameter $g_{\mathrm{pp}}^{T=1}$ can be adjusted such that the Fermi NME of $2 \nu \beta \beta$ decay vanishes leading to the needed restoration of the isospin symmetry for the $2 \nu \beta \beta$ decay. Keeping the obtained value of $g_{\mathrm{pp}}^{T=1}$ one can independently vary $g_{\mathrm{pp}}^{T=0}$ in order to fix its value by the data on $2 \nu \beta \beta$-decay half-lives. The obtained values of the two parameters can now, in turn, be used in the calculation of the $0 v \beta \beta$ NMEs. Hence, in this two-stage fit the maximum amount of information on $2 \nu \beta \beta$ decay can be utilized to produce an optimized model Hamiltonian for the description of the $0 \nu \beta \beta$ decay. This two-step fit was later used in [64] for $p n$ QRPA-based $0 v \beta \beta$-decay calculations and, about the same time, in [65] the isospin-restoration scheme was adopted for the IBM- $2 \beta \beta$ framework.

\section{RESULTS FOR THE REDUCED HALF-LIVES OF THE $0 v \beta \beta$-DECAY CANDIDATES}

In $[63,64]$ the two-step fit was quite limited: the experimental $2 \nu \beta \beta$ NME was extracted only for the free value $g_{A}^{\text {free }}=1.27$ and for a moderately quenched value $g_{A}=1.0$ for the axial-vector coupling constant. This corresponds to two values of $g_{\mathrm{pp}}^{T=0}$ which were used in the $0 \nu \beta \beta$-decay calculations. In this article the calculations of [64] are extended to include all values of $g_{A}$ below its free value. The adopted single-particle bases, pairing parameters, and values of the isovector parameter $g_{\mathrm{pp}}^{T=1}$ are taken from [64] (see, e.g., Table I of [64]) and the details are not repeated here. Now, instead of the two values of the $0 \nu \beta \beta$ NMEs of [64], one obtains a continuous sequence of $0 \nu \beta \beta$ NMEs as a function of $g_{A}$, as shown for five exemplary cases in Fig. 2.

A general feature of the $0 \nu \beta \beta$ NMEs, visible in Fig. 2, is that they grow in magnitude with diminishing value of $g_{A}$. This stems from the structure (3) of the $0 \nu \beta \beta$ NME: The Fermi and tensor NMEs are essentially independent of the value of $g_{A}$, whereas the Gamow-Teller NME grows in magnitude as $g_{A}$ decreases and, at the same time, $g_{\mathrm{pp}}^{T=0}$ decreases (the magnitude of the computed $2 \nu \beta \beta$ NME increases when $g_{\mathrm{pp}}^{T=0}$ decreases, necessitating a decrease in the value of $g_{A}$ in the fit procedure). Thus the $g_{A}$ dependence of the Gamow-Teller NME is not explicit but induced by the $g_{\mathrm{pp}}$ dependence of the nuclear wave functions and the adopted fit prescription of the $g_{\mathrm{pp}}^{T=0}$ parameter. The Fermi NME adds coherently with the Gamow-

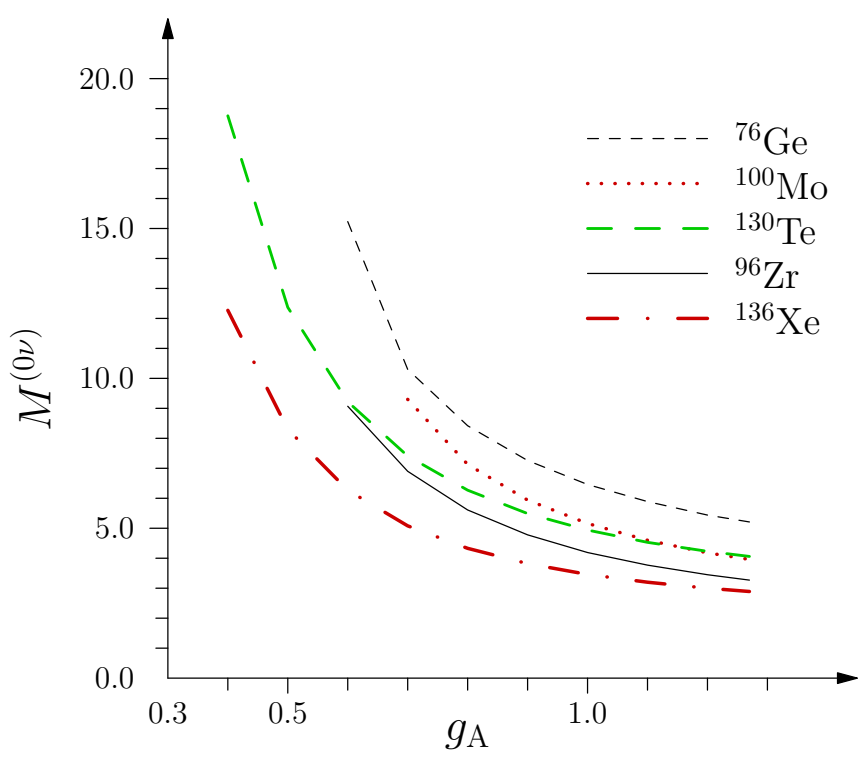

FIG. 2. Values of the $0 v \beta \beta$ NME (3) as functions of $g_{A}$ for the decays of ${ }^{76} \mathrm{Ge},{ }^{96} \mathrm{Zr}$, ${ }^{100} \mathrm{Mo},{ }^{130} \mathrm{Te}$, and ${ }^{136} \mathrm{Xe}$.

Teller one since the NMEs have opposite sign. In addition, the magnitude of the combination $\left(g_{\mathrm{V}} / g_{\mathrm{A}}\right)^{2} M_{\mathrm{F}}^{(0 v)}$ grows large, comparable to the magnitude of the Gamow-Teller NME, since the prefactor $\left(g_{\mathrm{V}} / g_{A}\right)^{2}$ grows rapidly with decreasing $g_{\mathrm{A}}$.

The maximum value of the $2 \nu \beta \beta$ Gamow-Teller NME is attained at $g_{\mathrm{pp}}^{T=0}=0$. This, in turn, means that there is a minimum value of $g_{A}$ for which the maximum NME can fit the $2 \nu \beta \beta$-decay half-life. This minimum value of the possible $g_{A}$ is an interesting byproduct of the two-stage fit and this lower limit of possible $g_{A}$ values is presented in Fig. 1 as a solid black line and in Table I in the second column. From Fig. 1 it is seen that the here obtained lower limit of $g_{A}$ is consistent with the thick green vertical bars of $g_{A}$ ranges obtained in $[41,42]$ and also consistent with the thin black vertical bars obtained in [36] for $A=100$ and $A=128$. For $A=116$ the analysis of [36] produces a larger value of $g_{A}$ than that obtained in $[41,42]$. This difference could be due to different experimental $\log f t$ values for the EC branch ${ }^{116} \mathrm{In} \rightarrow{ }^{116} \mathrm{Cd}$ used in the different

TABLE I. The obtained minimum values of $g_{A}$, the computed maximum and minimum values of the reduced half-life $F^{(0 v)}$, and their ratio for the studied $0 v \beta \beta$ systems.

\begin{tabular}{lcccc}
\hline \hline System & \multicolumn{2}{c}{$F^{(0 v)}$} & $F^{(0 v)}(\min )$ & Ratio \\
\cline { 2 - 4 } & $g_{A}(\min )$ & $F^{(0 v)}(\max )$ & & \\
\hline$A=76$ & 0.59 & $4.4 \times 10^{24}$ & $1.6 \times 10^{24}$ & 2.7 \\
$A=82$ & 0.56 & $2.1 \times 10^{24}$ & $7.0 \times 10^{23}$ & 3.0 \\
$A=96$ & 0.52 & $2.2 \times 10^{24}$ & $4.5 \times 10^{23}$ & 4.9 \\
$A=100$ & 0.70 & $7.9 \times 10^{23}$ & $4.0 \times 10^{23}$ & 2.0 \\
$A=116$ & 0.62 & $1.1 \times 10^{24}$ & $3.6 \times 10^{23}$ & 3.1 \\
$A=128$ & 0.38 & $3.4 \times 10^{25}$ & $7.7 \times 10^{24}$ & 4.4 \\
$A=130$ & 0.35 & $2.1 \times 10^{24}$ & $4.3 \times 10^{23}$ & 4.9 \\
$A=136$ & 0.36 & $4.7 \times 10^{24}$ & $8.2 \times 10^{23}$ & 5.7 \\
\hline \hline
\end{tabular}




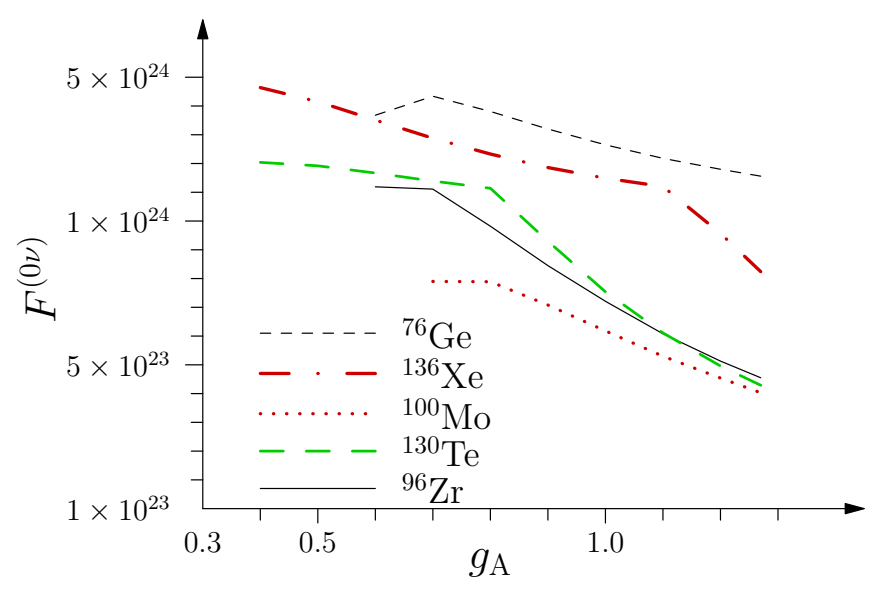

FIG. 3. Values of the reduced half-lives $F^{(0 v)}$ as functions of $g_{A}$ for the decays of ${ }^{76} \mathrm{Ge},{ }^{96} \mathrm{Zr},{ }^{100} \mathrm{Mo},{ }^{130} \mathrm{Te}$, and ${ }^{136} \mathrm{Xe}$.

analyses, $[41,42]$ using more recent data for this decay branch than [36] (see [42] for details).

There is a notable tension with the results of $[36,41,42]$ and the the Gamow-Teller $\beta$-decay analysis (hatched region) in the interval $A=98-116$. The basic difference between the Gamow-Teller analyses of [43-45] and the analyses of $[36,41,42]$ is that the latter exploit the half-life data on $2 \nu \beta \beta$ decays and the former not. It is more appropriate to include the $2 \nu \beta \beta$ data if $0 \nu \beta \beta$ properties are to be predicted since the $2 v \beta \beta$-decay observables are the closest to the $0 v \beta \beta$-decay observables. Thus, from the point of view of the present work it is preferable to compare the results with those obtained in $[36,41,42]$.

As mentioned earlier, one would expect that the reduced half-life $F^{(0 v)}$ of Eq. (2) would grow enormously with increasing value of $g_{A}$ since it is proportional to $\left(g_{A}\right)^{-4}$. This is in the very core of the large impact caused by the work by Barea et al. in [35]. Since now all the ingredients of Eq. (2) in the two-stage fit have been produced, it is possible to plot the values of $F^{(0 v)}$ as functions of $g_{A}$. This has been done in Fig. 3 for the exemplary cases whose NMEs were presented in Fig. 2. Inspection of Fig. 3 confirms a rather unexpected stunning result which is the reason for this article: The reduced half-lives are not much affected by the large variation in the values of $g_{A}$. The maximum and minimum values of this half-life, as also their ratios, are listed in Table I. From the table one can see that the ratio of the reduced (and also actual) half-lives ranges from 2.0 (the decay of ${ }^{100} \mathrm{Mo}$ ) to 5.7 (the decay of ${ }^{136} \mathrm{Xe}$ ). This is extremely good news for the present and future $0 \nu \beta \beta$-decay experiments since even the smallest values of $g_{A}$, obtained in some nuclear models, do not ruin the sensitivity of the experiments.

\section{CONCLUSIONS}

In conclusion, in this article it is shown by using a two-stage fit procedure to two-neutrino $\beta \beta$-decay data in the $p n \mathrm{QRPA}$ nuclear-theory framework that even the very strongly quenched values of the axial-vector coupling constant, obtained in many theoretical analyses, decrease the sensitivity of the present and future neutrinoless $\beta \beta$-decay experiments only by reduction factors $R_{1}=2-6$, depending on the decaying nucleus. This is in strong contrast with the possible huge values of the usually assumed simple-minded reduction factor $R_{2}=\left(g_{A}^{\text {free }} / g_{A}^{\text {eff }}\right)^{4}$, containing the ratio of the free and effective values of the axial-vector coupling constant: values of $R_{2} \approx 300$ are reachable in the worst scenarios proposed. This means that the knowledge of the effective value of the axial-vector coupling constant is not crucial for the neutrinoless $\beta \beta$-decay experiments from the point of view of experimental sensitivity.

The reason behind the moderate reduction in the experimental sensitivity is the strong dependence of the $\beta \beta$-decay NME on the value of the axial-vector coupling constant through the two-step fit procedure exploiting the two-neutrino $\beta \beta$-decay data. This mechanism is behind the important new finding that is communicated here, offering much better perspectives for detecting the neutrinoless $\beta \beta$ decay. Thus, the presently obtained results are extremely important for the community of $\beta \beta$ experiments, as also for nuclear, neutrino, and particle physics in general.

\section{ACKNOWLEDGMENTS}

This work has been partially supported by the Academy of Finland under the Finnish Centre of Excellence Programme 2012-2017 (Nuclear and Accelerator Based Programme at JYFL).
[1] J. Suhonen and O. Civitarese, Phys. Rep. 300, 123 (1998).

[2] J. D. Vergados, H. Ejiri, and F. Šimkovic, Rep. Prog. Phys. 75, 106301 (2012).

[3] J. D. Vergados, H. Ejiri, and F. Šimkovic, Int. J. Mod. Phys. E 25, 1630007 (2016).

[4] A. S. Barabash, Phys. Rev. C 81, 035501 (2010).

[5] A. S. Barabash, Nucl. Phys. A 935, 52 (2015).

[6] M. Agostini et al. (GERDA Collaboration), Nature 544, 47 (2017).

[7] C. Cuesta et al. (MAJORANA Collaboration), AIP Conf. Proc. 1686, 020005 (2015).
[8] R. Arnold et al. (NEMO-3 Collaboration), JETP Lett. 80, 377 (2004).

[9] R. Hodák et al. (SuperNEMO Collaboration), AIP Conf. Proc. 1686, 020012 (2015).

[10] K. Fushimi et al. (MOON Collaboration), J. Phys. Conf. Series 203, 012064 (2010).

[11] J. Argyriades et al. (NEMO-3 Collaboration), Nucl. Phys. A 847, 168 (2010).

[12] R. Arnold et al. (NEMO-3 Collaboration), Phys. Rev. D 92, 072011 (2015).

[13] H. Park et al. (AMoRE Collaboration), AIP Conf. Proc. 1686, 020016 (2015). 
[14] T. B. Becker et al. (LUMINEU Collaboration), Astropart. Phys. 72, 38 (2016).

[15] D. R. Artusa et al. (CUPID Collaboration), Phys. Lett. B 767, 321 (2017).

[16] R. Arnold et al. (NEMO-3 Collaboration), Phys. Rev. D 95, 012007 (2017).

[17] F. A. Danevich, et al. (AURORA Collaboration), J. Phys. Conf. Series 718, 062009 (2016).

[18] S. Zatschler et al. (COBRA Collaboration), AIP Conf. Proc. 1686, 020027 (2015).

[19] K. Alfonso et al. (CUORE Collaboration), Phys. Rev. Lett. 115, 102502 (2015).

[20] S. Andringa et al. (SNO+ Collaboration), Adv. High Energy Phys. 2016, 6194250 (2016).

[21] J. B. Albert et al. (EXO-200 Collaboration), Nature 510, 229 (2014).

[22] A. Gando, Y. Gando, T. Hachiya, A. Hayashi, S. Hayashida, H. Ikeda, K. Inoue, K. Ishidoshiro, Y. Karino, M. Koga, S. Matsuda, T. Mitsui, K. Nakamura, S. Obara, T. Oura, H. Ozaki, I. Shimizu, Y. Shirahata, J. Shirai, A. Suzuki, T. Takai, K. Tamae, Y. Teraoka, K. Ueshima, H. Watanabe, A. Kozlov, Y. Takemoto, S. Yoshida, K. Fushimi, T. I. Banks, B. E. Berger, B. K. Fujikawa, T. ODonnell, L. A. Winslow, Y. Efremenko, H. J. Karwowski, D. M. Markoff, W. Tornow, J. A. Detwiler, S. Enomoto, and M. P. Decowski (KamLAND-Zen Collaboration), Phys. Rev. Lett. 117, 082503 (2016).

[23] J. Martin-Albo et al. (NEXT-100 Collaboration), J. High Energy Phys. 05 (2016) 159.

[24] X. Chen et al. (PandaX-III Collaboration), Sci. China Phys. Mech. Astron. 60, 061011 (2017).

[25] J. Engel and J. Menéndez, Rep. Prog. Phys. 80, 046301 (2017).

[26] J. Kotila and F. Iachello, Phys. Rev. C 85, 034316 (2012).

[27] C. Patrignani et al. (Particle Data Group), Chin. Phys. C 40, 100001 (2016).

[28] B. A. Brown, W. Chung, and B. H. Wildenthal, Phys. Rev. Lett. 40, 1631 (1978).

[29] W. T. Chou, E. K. Warburton, and B. A. Brown, Phys. Rev. C 47, 163 (1993).

[30] B. A. Brown and B. H. Wildenthal, Annu. Rev. Nucl. Part. Sci 38, 29 (1988).

[31] G. Martínez-Pinedo, A. Poves, E. Caurier, and A. P. Zuker, Phys. Rev. C 53, R2602 (1996).

[32] T. Siiskonen, M. Hjorth-Jensen, and J. Suhonen, Phys. Rev. C 63, 055501 (2001).

[33] V. Kumar, P. C. Srivastava, and H. Li, J. Phys. G 43, 105104 (2016).

[34] I. S. Towner, Phys. Rep. 155, 263 (1997).
[35] J. Barea, J. Kotila, and F. Iachello, Phys. Rev. C 87, 014315 (2013).

[36] A. Faessler et al., J. Phys G 35, 075104 (2008); arXiv:0711.3996v1 [nucl-th].

[37] P. Vogel and M. R. Zirnbauer, Phys. Rev. Lett. 57, 3148 (1986).

[38] O. Civitarese, A. Faessler, and T. Tomoda, Phys. Lett. B 194, 11 (1987).

[39] A. Juodagalvis and D. J. Dean, Phys. Rev. C 72, 024306 (2005).

[40] E. Caurier, F. Nowacki, and A. Poves, Phys. Lett. B 711, 62 (2012).

[41] J. Suhonen and O. Civitarese, Phys. Lett. B 725, 153 (2013).

[42] J. Suhonen and O. Civitarese, Nucl. Phys. A 924, 1 (2014).

[43] H. Ejiri and J. Suhonen, J. Phys. G. 42, 055201 (2015).

[44] P. Pirinen and J. Suhonen, Phys. Rev. C 91, 054309 (2015).

[45] F. F. Deppisch and J. Suhonen, Phys. Rev. C 94, 055501 (2016).

[46] N. Yoshida and F. Iachello, Prog. Theor. Exp. Phys. 2013, 043D01 (2013).

[47] J. Suhonen and O. Civitarese, J. Phys. G 39, 085105 (2012).

[48] J. Toivanen and J. Suhonen, Phys. Rev. Lett. 75, 410 (1995).

[49] C. M. Raduta and A. A. Raduta, Phys. Rev. C 82, 068501 (2010).

[50] E. Caurier, G. Martinez-Pinedo, F. Nowacki, A. Poves, and A. Zuker, Rev. Mod. Phys. 77, 427 (2005).

[51] J. Barea and F. Iachello, Phys. Rev. C 79, 044301 (2009).

[52] T. R. Rodríguez and G. Martínez-Pinedo, Phys. Rev. Lett. 105, 252503 (2010).

[53] P. K. Rath, R. Chandra, K. Chaturvedi, P. K. Raina, and J. G. Hirsch, Phys. Rev. C 82, 064310 (2010).

[54] J. M. Yao, L. S. Song, K. Hagino, P. Ring, and J. Meng, Phys. Rev. C 91, 024316 (2015).

[55] N. L. Vaquero, T. R. Rodríquez, and J. L. Egido, Phys. Rev. Lett. 111, 142501 (2013).

[56] J. Suhonen and O. Civitarese, J. Phys. G 39, 124005 (2012).

[57] R. A. Sen'kov and M. Horoi, Phys. Rev. C 88, 064312 (2013).

[58] O. Civitarese, H. Müther, L. D. Skouras, and A. Faessler, J. Phys. G 17, 1363 (1991).

[59] V. A. Rodin, A. Faessler, F. Šimkovic, and P. Vogel, Nucl. Phys. A 766, 107 (2006).

[60] M. Kortelainen and J. Suhonen, Phys. Rev. C 75, 051303(R) (2007)

[61] M. Kortelainen and J. Suhonen, Phys. Rev. C 76, 024315 (2007).

[62] F. Šimkovic, A. Faessler, V. A. Rodin, P. Vogel, and J. Engel, Phys. Rev. C 77, 045503 (2008).

[63] F. Šimkovic, V. Rodin, A. Faessler, and P. Vogel, Phys. Rev. C 87, 045501 (2013).

[64] J. Hyvärinen and J. Suhonen, Phys. Rev. C 91, 024613 (2015).

[65] J. Barea, J. Kotila, and F. Iachello, Phys. Rev. C 91, 034304 (2015) 